\title{
Soft X-Ray Band Spectra and Their Relationship to the Density of States *
}

\author{
G. A. Rooke \\ Metallurgy Department, University of Strathclyde, Glasgow ${ }^{1}$ \\ (October 10, 1969)
}

\begin{abstract}
The paper concentrates on the similarities and differences between the one-electron spectrum and the density of states; many-body effects, although important, are listed but they are not considered in detail. It is shown that the only reliable information about the density of states that can be obtained from soft x-ray spectroscopy are the energies of the Fermi surface and the van-Hove singularities, although the shape of the density of states can be derived indirectly from the energies of the van-Hove singularities.

It is the differences between the density of states and the one-electron spectra that may prove to be most important. These differences can give information about the symmetry and the local nature of the screening electrons. This is particularly interesting when studying alloys.

The $\mathrm{Li} \mathrm{K}$, the $\mathrm{Al} \mathrm{L}_{23}$ and the $\mathrm{Zn} \mathrm{L}_{3}$ spectra are given as examples which illustrate the above arguments. Finally, a brief discussion on the soft x-ray spectra from the Al-Mg system show how the results may be used to study alloys.
\end{abstract}

Key words: Alloys; auger transitions; density of states; many-body interactions; plasmons; singularities; soft x rays.

\section{Introduction}

Before commencing my discussion, I would like to state our objectives in attempting to measure the density of states: they are

(a) to compare experiments that are in some way related to the density of states;

(b) to derive some information about concepts of a more fundamental nature, the band structure, the effective potential, etc., and

(c) to attempt to predict the properties of other metals and alloys.

It is important to bear these in mind, as sometimes our objectives can be achieved more directly by not making use of the density of states.

It will be remembered that when transitions involving atomic core states occur, x-rays may be emitted or absorbed. The $x$-rays have an energy equal to the energy difference between the two states involved in the transition. If one of the states lies in the valence band or the conduction band, a band spectrum is produced

*An invited paper presented at the 3d Materials Research Symposium, Electronic Density of States, November 3-6, 1969, Gaithersburg, Md.

'Present address: Ferranti Ltd., Western Road, Bracknell, Berks, U.K. (see fig. 1). Experimentally, better energy resolution is obtained from the band spectra with lower energies; these are called soft x-ray band spectra. Although this paper specifically discusses soft x-ray spectra, the concepts are applicable to all band spectra.

For an introductory review of soft x-ray band emission spectroscopy, I recommend either Skinner [1] or Tomboulian [2]. An excellent bibliography of Yakowitz and Cuthill [3] reviews the literature up to 1961. Some of the most recent work in the field is described in a conference proceedings edited by Fabian [4]. All of these omit very interesting Russian work for which no comprehensive review is known to me.

\section{Many-Body Interactions}

The many-body interactions are treated in this paper as uninteresting complications that tend to hide the information we are seeking. Each interaction is considered as a perturbation on the one-electron spectrum.

The interactions between individual electrons create two types of perturbations on the spectra, the excited initial and final states, which directly affect the spectra, and exchange and correlation, which affect the spectra 


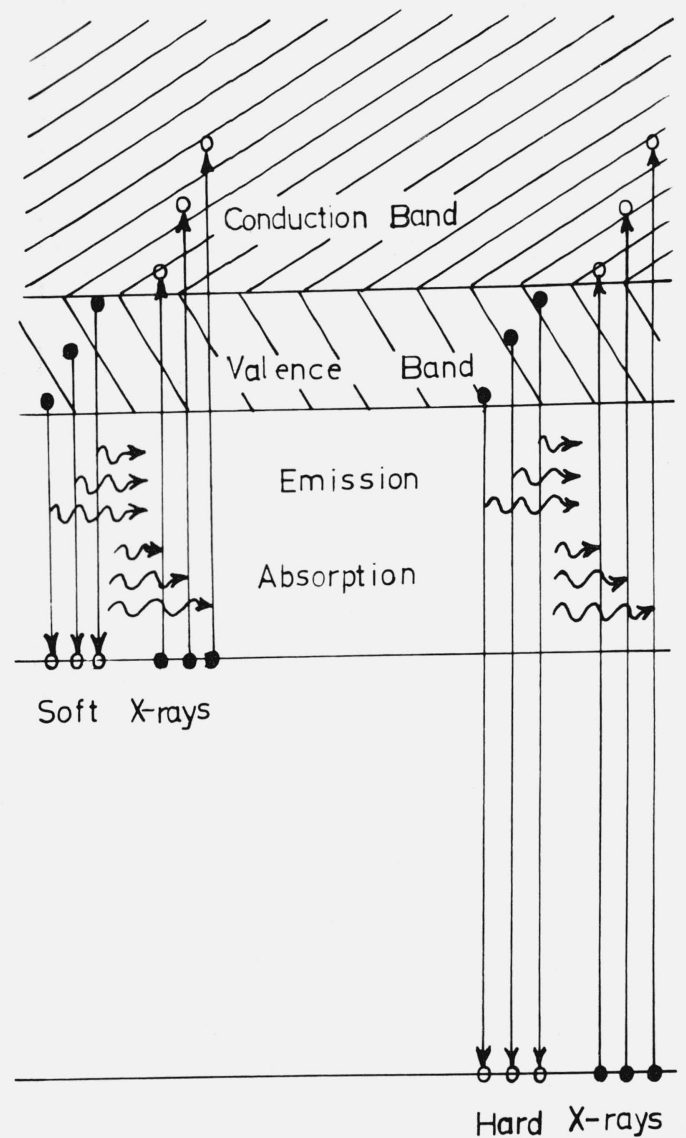

Figure 1. One-electron energy-level diagram showing $x$-ray transitions.

through their effects on the band structure. The exchange and correlation only cause trouble when comparing uncorrected one-electron band-structures with experimental results [5]. They are normally allowed for by theoreticians and are only important to experimentalists when they are measuring many-body effects or when they are trying to derive the uncorrected bandstructures.

Excited states occur whenever electron transitions occur. For all radiating transitions, two single-particle excitations are involved; in emission processes both the initial and the final states are normally excited while in absorption processes the initial state is normally unexcited and the final state contains two single-particle excitations. Each excitation affects the spectrum through its lifetime and through its perturbing effects on the unexcited one-electron spectrum. Three types of one-electron excitation can occur and their effects on the spectra are discussed separately.

First, the hole in the core state perturbs the valence electrons, so that the intensity at the Fermi surface is reduced for $\mathrm{K}$ spectra (transitions to ls-states) and enhanced for $\mathrm{L}_{23}$ spectra (transition to 2p-states) [6]. The lifetime of the hole in the core state causes the spectrum to be broadened because of the uncertainty in its energy. If this lifetime is known, it is possible to correct for this broadening [7]. The hole in the core state can only affect spectra that involve x-rays.

Second, a hole in the valence band can be filled by an electron in the same band, provided another valenceband electron is excited into the conduction band, thereby conserving energy; this interaction is called the Auger process. Because of this, there are both holes and excited electrons with energies close to the Fermi energy and this raises the possibility of excitons occurring in the metal. Also, Auger processes shorten the lifetime of the hole and this broadens the spectra considerably. Because many more electrons are capable of filling holes near the bottom of the band than those near the top of the band, the lifetime of a hole near the bottom of the band is shorter and the spectrum is broadened more near the bottom of the band than near the top. It has not yet proved possible to remove the Auger broadening from spectra, because the broadening is not constant throughout the band. Auger broadening is important to any spectroscopy involving the absorption of the ultraviolet light, to x-ray emission spectroscopy and to ion-neutralization spectroscopy.

Third, the scattering of electrons excited into the conduction band is important to any process involving the absorption of radiation. This scattering is very similar to the Auger process; an excited electron falls to an energy level closer to the Fermi energy, giving its energy to another electron which is excited out of the valence band and into the conduction band. The remaining holes and the scattered electrons could form excitons. For any electron emission spectroscopy the scattered electrons can contribute to the spectra and they enhance the spectra near the Fermi edge. In general, the lifetime of the excited states decreases as their energy increases, so that the resultant broadening is not constant throughout the band and is exceedingly difficult to remove.

For each type of optical spectroscopy discussed at this conference, two of these three types of one-electron excitations are involved and for ion-neutralization spectroscopy, four excitations occur.

The first two types of excitations are involved in soft $x$-ray band emission spectroscopy. The perturbations of the valence band, near the Fermi edge, by the hole in the core state is possibly the cause of the drop in intensity near the Fermi edge in the lithium K spectrum and the cause of the small pip near the Fermi edge in the sodium $\mathrm{L}_{23}$ spectrum [6]. It probably contributes more 
to the shape of all light metal spectra than to the heavy metal spectra, because the hole represents a larger proportion of the core electrons in the light metals. The core broadening prevents the detection of spectra resulting from transitions to $2 \mathrm{~s}, 3 \mathrm{~s}$, etc. core states and it also is important in changing the shape of the spectra from heavy metals; the lifetime of the excited state is considerably reduced because of competing non-radiative transitions. Auger broadening is responsible for the low-energy tails in the spectra from metals and this introduces uncertainty into the measurement of the bandwidths.

For other spectroscopic techniques, these excitations are also highly important, their importance depending on both the technique used and the metal examined.

Besides exciting a single electron state, it is also possible to excite the electrons collectively into plasma oscillations. When this occurs, together with the emission of an x-ray photon, the photon loses enough energy to create the plasmon and a satellite spectrum is formed at lower energies [8,9]. Interactions between individual electrons and the plasmons, which are called plasmarons [10], have a measurable effect on the plasmon satellite [11] but not on the parent band.

\section{The Density of States and Band Spectra}

In order to discuss the relationship between the density of states and the spectra, it is assumed in the remainder of this paper that the effects of the manybody interactions can be corrected for. The word "spectrum" then implies "one-electron spectrum that would result if such corrections were made"; this spectrum has the same width as the valence band, its shape is related to the density of states but it is affected by the transition probabilities.

The similarities between the density of states, N(E), and the one-electron spectrum divided by the cube of the radiation frequency, $\frac{I(E)}{\nu^{3}}$, are seen by comparing the following equations:

$$
\begin{gathered}
N(E) \propto \int_{s} d^{2} \mathbf{k} \frac{1}{\nabla_{\mathbf{k}} E} \\
I(E) / \nu^{3} \propto \int_{s} d^{2} \mathbf{k} \frac{1}{\nabla_{\mathbf{k}} E}\left|\int \psi_{\mathbf{k}} \nabla_{\mathbf{x}} \psi_{f} d \tau\right|^{2}
\end{gathered}
$$

where $\mathrm{E}$ is the energy of the state with wave vector $\mathbf{k}$ and the integral is taken over the surface, $s$, of constant energy, E. For the density of occupied states and for the emission spectrum, the integral is taken over the region of $\mathbf{k}$ space that lies inside the Fermi surface, while, for the density of unoccupied states and for the absorption spectrum, the integral is taken over the region of $\mathbf{k}$ space that lies outside the Fermi surface. The square of the modulus of the matrix element, $\int \psi_{\mathbf{k}} \nabla_{\mathbf{x}} \psi_{f} d \tau$, is the transition probability, whose properties determine the difference between the two functions; the dipole approximation has been assumed.

A striking similarity between the two functions occurs because the integrations are terminated at the Fermi surface; this produces the Fermi edges in the functions. Unfortunately, for some heavy metals, the core-state broadening and the transition probabilities combine to make the Fermi edge almost impossible to measure.

The Brillouin zone effects are also common to both functions; at certain points of high symmetry on the Brillouin zone boundaries, the gradient, $\nabla_{\mathbf{k}} \mathrm{E}$, is zero and van-Hove singularities exist at corresponding points in the two functions. Certain van-Hove singularities are of particular interest; in particular the energy of the bottom of the band can be used to estimate the bandwidth and the energy of the top and bottom of the $\mathrm{d}$-band give the width of this band and its relation to the Fermi energy, both of which are of considerable theoretical interest.

Because the matrix element is $\mathbf{k}$-dependent, it is not possible to remove the transition probabilities from inside the integral in eq (2). Hence, it is not possible to write the intensity as a product of the density of states and the average transition probability and, even if the transition probabilities are fully known, it is not possible to remove their effects from the spectra. Rooke [12] has shown that the k-dependence of the matrix element is large, sometimes changing it from nearly 1 to nearly 0 on the same constant energy curve, so that errors created by removing the matrix element from the integral can be serious.

From the above considerations it can be seen that the detailed density of states cannot be directly derived by using soft $\mathrm{x}$-ray spectroscopy; or by using any form of spectroscopy, for that matter. Sometimes a spectrum and the density of states will have similar shapes and they will certainly have the Fermi edge and some vanHove singularities in common. If the spectrum changes under different experimental conditions, such as heat or pressure, it may be possible to assume that the changes will be entirely due to the density of states and to obtain a little more information about the density of states in this way. This technique has been used to find the effect of the Fermi-Dirac statistics on the density of states, by heating the target (1) and to find the effect of 
alloying on the density of states. A more sophisticated version of this approach has just been developed by J. H. Willens in Bell Laboratories [13]. In another method for obtaining more information about the density of states, one derives a band structure that fits the measured van-Hove singularities and then uses this to calculate the density of states. However, as Fermi surface techniques are capable of making fine distinctions between band structures, they can also be used to obtain densities of states which are often more accurate than those obtained from soft x-ray spectroscopy. Even though it is only possible to obtain limited information about the density of states, this information is often very interesting. However, it is often even more interesting to examine the information that can be derived by studying the effect of the transition probabilities on the spectra. This information is discussed in the next section.

\section{The Transition Probability and the Band Spectra}

The differences between the functions defined in eqs (1) and (2) are determined by the matrix element

$$
\int \psi_{\mathbf{k}} \nabla_{\mathbf{x}} \psi_{f} d \tau
$$

The integration is taken over all real space and $\nabla_{\mathbf{x}}$ is the component of the real-space gradient-operator in the $\mathbf{x}$ direction.

$\psi_{f}$ is the wavefunction of the core state and, because of its high symmetry, it is a good approximation to express it as

$$
\psi_{f}(r, \theta, \phi)=R_{n^{\prime}}(r) Y_{l^{\prime}}(\theta) e^{i m^{\prime} \phi}
$$

where $R_{n^{\prime}}(r)$ is a radical function, $Y_{l^{\prime}}(\theta)$ is a Legendre polynomial and $\mathrm{n}^{\prime}, \mathrm{l}^{\prime}$ and $\mathrm{m}^{\prime}$ are the usual quantum numbers. The radial function has appreciable magnitude only near the center of the atom and the state is localized. Because only one Legendre polynomial is involved, the state can be labeled by atomic notation; $1 \mathrm{~s}$, $2 \mathrm{p}, 3 \mathrm{~d}$, et $c$.

$\psi_{\mathbf{k}}$ is the wavefunction of a valence state and it may be expanded as a series of spherical harmonies

$$
\psi_{\mathbf{k}}=\sum_{l, m} C_{l}(\mathbf{k}) a_{l, \mathbf{k}}(r) Y_{l}(\theta){ }^{i m \phi}
$$

Normally, only the first three values of $l$ are important. The radial terms are roughly constant throughout real space so that the state is shared by all atoms and is not localized like the core states.
Substituting these expansions of the wavefunctions into the matrix elements gives

$$
\begin{aligned}
\int \psi_{\mathbf{k}} \nabla_{\mathbf{x}} \psi_{f} d \tau \propto \sum_{l, m} C_{l}(\mathbf{k}) \times \int R_{n^{\prime}}(r) a_{l, \mathbf{k}}(r) r^{3} d r \\
\times \int Y_{l^{\prime}}(\theta) Y_{l}(\theta) f\left(\sin _{\cos } \theta\right) d \theta \times \int e^{i\left(m-m^{\prime}\right) \phi g\left(\sin _{\cos } \phi\right) d \phi}
\end{aligned}
$$

where $f$ and $g$ are functions whose form depends on the polarization. Each of these four terms contributes a distinct characteristic to the transition probability. The k-dependence of the first two terms, as shown in the last section, prevents the direct derivation of the density of states from the spectra.

The third term is zero unless

$$
l=l^{\prime} \pm 1
$$

This selection rule is particularly important for $\mathrm{x}$-ray transitions, because the core state involved has a well defined symmetry described by a single spherical harmonic. If the core state is an s-state, only p-like states can make the transition to it. Similarly, only s-like and d-like states can make the transition to a p-state.

This selection rule enables us to describe states in terms of partial densities of states; the density of sstates, the density of p-states and the density of $\mathrm{d}$ states. K spectra (transitions to the s-state) give approximate estimates of the density of $p$-states, while $L_{23}$ or $M_{23}$ spectra (transitions to the $2 p$ or $3 p$ states respectively) give approximate estimates of the density of $s$ and d states.

Because these partial densities of states only approximate to the shape of the spectra, it is not possible to add them and to derive a meaningful density of states. However, this symmetry dependence of $x$-ray band spectra makes them unique in the information that they can reveal. This is particularly interesting in the case of alloys, for which this characteristic of x-ray spectra should be fully exploited to determine the nature of the screening charges. Further, it is the authors unsubstantiated view, that the extended fine-structure occurring in absorption spectra can also be attributed to this selection rule.

Besides creating gross features in the spectra, this selection rule modifies the magnitudes of the van-Hove singularities. The symmetry of the crystal dictates that the states associated with points of high symmetry in the Brillouin zone will have high symmetry themselves. Thus, some of these states are almost entirely p-like while others are s-like or $\mathrm{s}$ and $\mathrm{d}$ like. If a state is entirely s-like, it will not contribute to a $\mathrm{K}$ spectrum and, if it 
is associated with a van-Hove singularity, that singularity will not be seen in the K spectrum. Similarly, as most states contain a mixture of symmetries, van-Hove singularities associated with states that are entirely p-like will be exaggerated in the $\mathrm{K}$ spectra. The reverse situation occurs for $\mathrm{L}_{23}$ or $\mathrm{M}_{23}$ spectra, for which p-like singularities will not be observed and $\mathrm{s}$ and d like singularities will be exaggerated.

The last term in eq (5) is dependent on the polarization of the radiation and it is non-zero if $m=m^{\prime} \pm 1$, but if the polarization is suitable it may also be non-zero if $\mathrm{m}=\mathrm{m}^{\prime}$. We therefore write the second selection rule as

$$
\mathrm{m}=\mathrm{m}^{\prime}= \pm 1 \text { or } 0
$$

This rule implies that not all the transitions described in the second last paragraph are allowed. For any one type of transition, say s to $p$, only a fraction of transitions are allowed and this fraction may vary throughout the band, thereby distorting the spectrum. However, this distortion may not be serious, as the fraction may be nearly constant throughout the band. Of course, the constant fraction for $\mathrm{d}$ to $\mathrm{p}$ type transitions will be different from that for $s$ to $p$ type transitions and, in fact, the fraction for these transitions can be shown to be about 2/5 of that for the $\mathrm{s}$ to $\mathrm{p}$ transitions. This means that the $\mathrm{L}_{23}$ or $\mathrm{M}_{23}$ spectra give an approximate measure of the density of s-states plus $2 / 5$ density of $\mathrm{d}$ states. This is one reason why the $\mathrm{L}_{23}$ and $\mathrm{K}$ spectra, when added together, do not give the full density of states.

The second term in eq (5) provides the spectra with some very interesting properties. The integral effectively takes a weighted average of a(r), using a weighting factor of $\mathrm{rR}_{n^{\prime}}(\mathrm{r})$. Because of the localized nature of $\mathrm{R}_{n^{\prime}}(\mathrm{r})$, this integral can be considered to take an average of $a(r)$ in the region near the core state of the emitting atom. This is exceedingly interesting when studying alloys, as the spectra from each component metal will sample the wavefunction in the region of that type of atom. These local properties are of great interest and are only obtainable by the spectroscopies involving $\mathrm{x}$-rays.

Finally, the degree of approximation involved in assuming that spectra are similar to the partial densities of states is discussed. Consider the case of the $\mathrm{K}$ spectrum, which samples p-states. Because the wavefunctions are normalized, the term $\int \psi_{\mathbf{k}}{ }^{2} \mathrm{~d} \tau$ may be inserted into eq (1) without changing it. This term may be written as

$$
\sum_{l}(2 l+1)^{2} C_{l}^{2}(\mathbf{k}) \int a_{l, \mathbf{k}}{ }^{2}(r) r^{2} d r
$$

and the density of p-states may be defined as

$$
N_{p}(E) \propto \int_{s}^{d^{2} \mathbf{k}} \frac{1}{\nabla_{\mathbf{k}} E} C_{l}^{2}(\mathbf{k}) \int a_{l, k}^{2}(r) r^{2} d r
$$

By replacing the last two terms in eq (5) by non-zero constants and restricting the summation to $l=1$, eq (2) may then be written as

$$
I_{k}(E) / v^{3} \propto \int^{d^{2} \mathbf{k}} \frac{1}{\nabla_{\mathbf{k}} E} C_{l}^{2}(\mathbf{k})\left|\int_{0} a_{l, \mathbf{k}}(r) r^{2} d r\right|^{2}
$$

where the zero on the radial integral indicates that it is now restricted to the region of the core state of the emitting atom. The final terms in these equations differ, one being the average of the square of a(r) and the other being the square of a local average of a(r). For the densities of s-states and of d-states and the corresponding spectra, the situation is complicated by the factor of $2 / 5$ and by cross terms, but the differences arise in the same way.

\section{The Spectra from Pure Metals}

It is shown above, that x-ray spectra sample only those wavefunctions that have specific symmetries and that lie near the core of the emitting atom. Apart from these restrictions, they tend to show some of the behavior of the density of states, particularly the Fermi edge and the van-Hove singularities. The spectra are broadened by the experimental resolution and by manybody effects and it is possible that these many-body effects also change the shape of the spectra near the Fermi edge. These ideas are now illustrated by three pure metal spectra; the $\mathrm{Li} \mathrm{K}$, the $\mathrm{Al} \mathrm{L}_{23}$ and the $\mathrm{Zn} \mathrm{L}_{3}$ emission spectra.

The Li K emission spectrum [14] is shown in figure 2. The Auger tail is immediately obvious but the Fermi

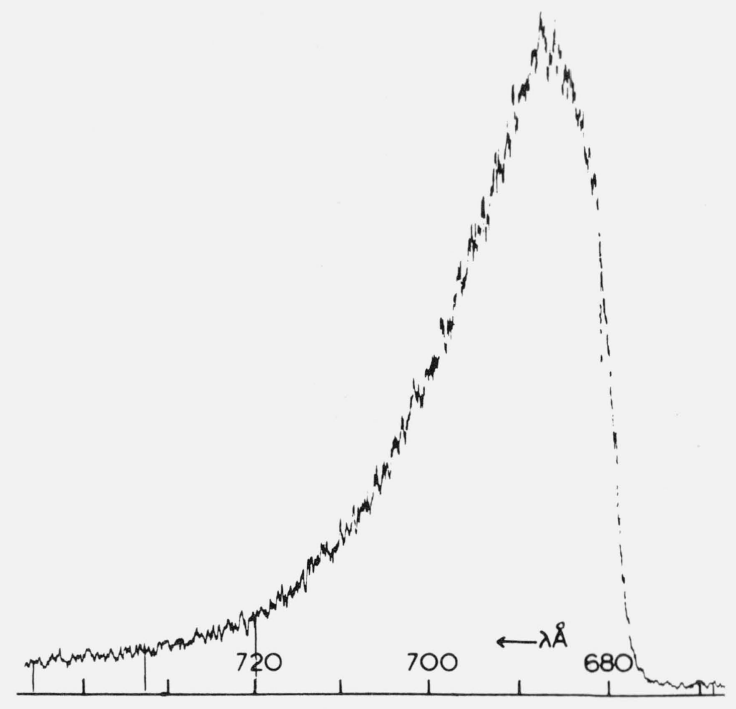

FIGURE 2. Lithium $K$ emission spectrum. Reproduced from Crisp and Williams [14]. 


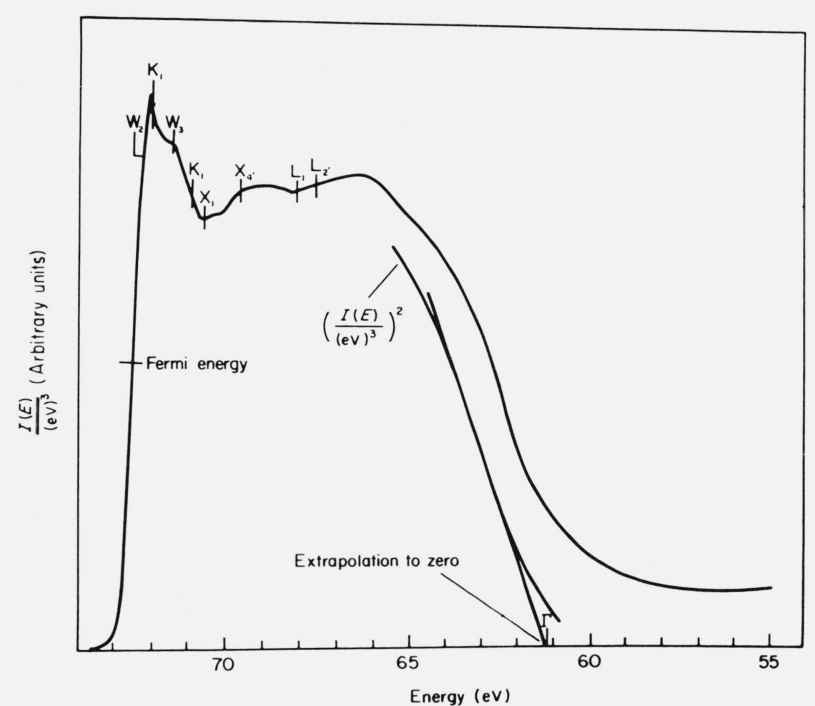

FigURE 3. Aluminum $L_{23}$ emission spectrum. Reproduced from Rooke [15].

edge at $\mathrm{eV}$ is not so obvious because of the fall in intensity towards the edge; this fall in intensity is attributed to the effect of the hole in the core state. Because of the low bandwidth, no van-Hove singularities occur below the Fermi edge. The shape of the density of states is almost certainly close to parabolic, but, because of the lack of p-like states near the bottom of the band, the spectrum does not reflect this; its intensity is considerably reduced near the bottom of the band.

The $\mathrm{Al} \mathrm{L}_{23}$ emission spectrum [15] is shown in figure 3. The Fermi edge and the Auger tail are particularly obvious and the van-Hove singularities appear as discontinuities in the top half of the band. It is seen that the p-like van-Hove singularities, $\mathrm{X}_{4^{\prime}}$ and $\mathrm{L}_{2^{\prime}}$, are much less obvious than the s-like singularities, $\mathrm{X}_{1}$ and $\mathrm{L}_{1}$. The bottom of the band rises sharply to a broad hump at about $66 \mathrm{eV}$; this reflects the parabolic rise of the density of states and the fact that the states near the bottom of the band are nearly all observed because they are mostly s-like. From $66 \mathrm{eV}$ to about $70 \mathrm{eV}$, the intensity

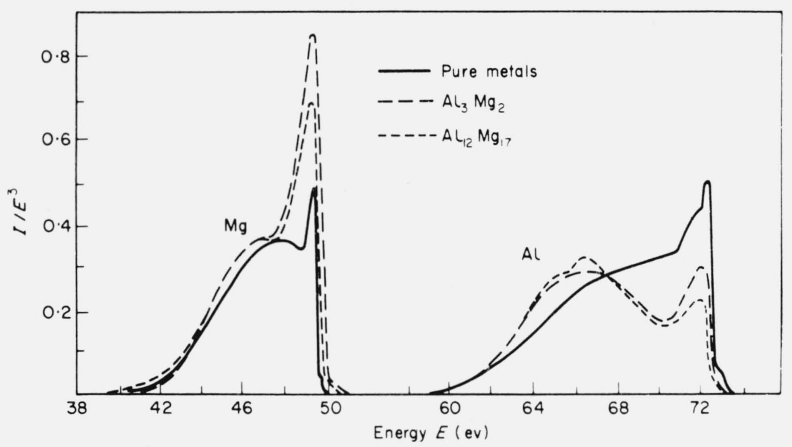

Figure 5. Magnesium $L_{23}$ and Aluminum $L_{23}$ emission spectra from Al-Mg alloys. Reproduced from [17].

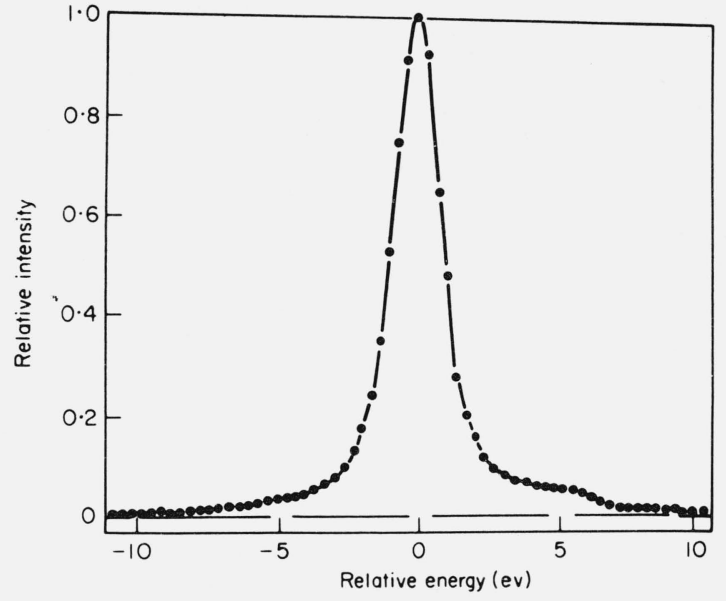

FIGURE 4. Zinc $L_{23}$ emission spectrum. Reproduced from Liefeld [16].

falls but then it rises again towards the Fermi edge. This occurs because the states change from being mostly s-like at the bottom of the band to being mostly p-like near the center of the band and then to having both $\mathrm{s}$ and $\mathrm{d}$ character near the top of the band.

The $\mathrm{Zn} \mathrm{L}_{3}$ emission spectrum [16] is shown in figure 4. This spectrum has been obtained after considerable effort in allowing for the various experimental errors that occur. The tall peak at the center of the spectrum is possibly the d-band while the sharp fall in intensity at about $+7 \mathrm{eV}$ may be the Fermi edge.

\section{The Spectra from Alloys}

Soft x-ray spectra are proving to be of considerable value for studying alloys and it is for this purpose that soft $x$-ray spectroscopy will be developed in the next few years. An excellent review of the work done in the West has been given by Curry [17], but the Russians have also done some interesting work, particularly on alloys containing d-band metals.

As an example the very interesting results from the $\mathrm{Al}-\mathrm{Mg}$ system are discussed. The $\mathrm{Mg} \mathrm{L}_{23}$ and $\mathrm{Al} \mathrm{L}_{23}$ emission spectra from two alloys are shown together with the pure metal spectra in figure 5 . At first sight, it is seen that the apparent bandwidths of the alloy spectra are not greatly different from those of the pure metal spectra. On alloying, the intensity of the $\mathrm{Mg}$ spectrum is relatively enhanced at the top of the band while the intensity of the bottom of the Al spectrum is relatively enhanced. The spectra have been carefully and independently checked by Dimond [18] and it is felt that the results are not due to clustering.

The following analysis produces some very interesting information. First, the width of the density of states must be at least as wide as the bandwidth of the $\mathrm{Al}$ spectrum; this fact is in contradiction with most theo- 
ries of alloys. Second, either the states at the bottom of the band must be highly localized around the aluminum atoms or they must have mostly s-symmetry in the region of the aluminum ions and mostly p-symmetry in the magnesium ions; the former alternative is the most likely. It is possible that the states are tunneling through a potential barrier in the region of the magnesium ions and can only contribute to the spectra when they are in the vicinity of the aluminum ions. Third, states that are localized near the aluminum ions tend to be relatively more s-like than the states at the same energy in the pure metal. For magnesium, the states near the bottom of the spectrum tend to be relatively more p-like, reflecting the fact that they are now not near the bottom of the band.

This example shows how useful it can be to sample electrons locally and with selected symmetries. Another example involving d-band metals is given later in the conference by Lindsay, W atson and Fabian.

\section{Acknowledgments}

The author wishes to acknowledge Dr. L. M. W atson, C. A. W. Marshall, and R. K. Dimond for their discussions and help in preparing this paper and also Dr. D.
J. Fabian and the Science Research Council for a Research Associateship (at the University of Strathclyde).

\section{References}

[1] Skinner, H. W. B., Phil. Trans. Roy. Soc. A239, 95 (1940).

[2] Tomboulian, D. H., Handbuch der Physik, XXX, 246 (1957).

[3] Yakowitz, H. and Cuthill, J. R., National Bureau of Standards (U.S.A.), Monograph 52,(1962).

[4] Soft X-ray Band Spectra and the Electronic Structure of Metals and Alloys, D. J. Fabian, ed., Academic Press, London (1968).

[5] Pines, D., Solid State Physics 1, 367 (1955).

[6] Mizuno, Y. and Ishikawa, K., J. Phys. Soc. Japan 25, 627 (1968).

[7] Liefeld, R. J., p. 133 in [4].

[8] Rooke, G. A., p. 3 in [4].

[9] See part 4 in [4].

[10] Hedin, L., p. 337 in [4].

[11] Cuthill, J. R., McAlister, A. J., Williams, M. L. and Dobbyn, R. C., p. 158 in [4].

[12] Rooke, G. A., J. Phys. Chem. 1, 767 (1968).

[13] Willens, J. H., Schreiber, H., Buehler, E., and Brasen, D., Phys. Rev. Letters 23, 413 (1969).

[14] Crisp, R. S. and Williams, S. E., Phil. Mag. (8)5, 525 (1960).

[15] Rooke, G. A., J. Phys. Chem. 1, 776 (1968).

[16] Liefeld, R., p. 149 in [4].

[17] Curry, C., p. 173 in [4].

[18] Dimond, R. K., Ph.D. Thesis, to be submitted to University of Western Australia, (1970). 